\title{
RETRACTED CHAPTER: Real-Life Facial Expression Recognition Systems: A Review
}

\author{
Samta Jain Goyal, Arvind K. Upadhyay, R. S. Jadon \\ and Rajeev Goyal
}

The editor has retracted this chapter [1] beca of significant overlap with an earlier published article by Benta and ida [2]. Authors Samta Jain Goyal, Arvind K. Upadhyay and Rajee G yal agree with this retraction. Author R. S. Jadon has not responded to ny correspondence from the publisher about this retraction.

[1] Goyal S. J., Upadhyay K., J don R. S., Goyal R. (2018) Real-Life Facial Expression Recognition S stem A Review. In: Satapathy S., Bhateja V., Das S. (eds) Smart Computi ig and Informatics. Smart Innovation, Systems and Technologies, vol 77. S inger, Singapore https://doi.org/10.1 7/978-981-10-5544-7_31.

[2] K.-I. Benta, M.-F. Vaida, "Towards Real-Life Facial Expression Recognition Systems," dv nces in Electrical and Computer Engineering vol. 15, no. 2, pp. 93-1U2, - 15, https://doi.org/10.4316/AECE.2015.02012. 